\title{
Interaction between Erythrocyte Phospholipid Fatty Acids Composition and Variants of Inflammation-Related Genes on Type 2 Diabetes
}

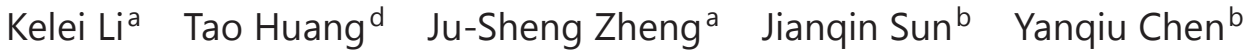 \\ Hua Xie ${ }^{b}$ Danfeng Xub Jianbo Wan ${ }^{c}$ Duo Li ${ }^{\mathrm{a}}$ \\ a Department of Food Science and Nutrition, Zhejiang University, Hangzhou, and \\ ${ }^{b}$ Clinical Nutrition Center, Huadong Hospital, Fudan University, Shanghai, PR China; \\ 'State Key Laboratory of Quality Research in Chinese Medicine, Institute of Chinese Medical \\ Sciences, University of Macau, Macao, SAR, PR China; ${ }^{d}$ Departments of Nutrition and \\ Epidemiology, Harvard School of Public Health, Boston, Mass., USA
}

\section{Key Words}

Type 2 diabetes mellitus · Single nucleotide polymorphism · Polyunsaturated fatty acids . Inflammation

\begin{abstract}
Aims: The present study aimed to investigate the interaction between erythrocyte phospholipid (PL) fatty acids and variants of inflammation-related genes in a Chinese population. Methods: A total of 622 patients with type 2 diabetes mellitus (T2DM) and 293 healthy subjects were included. Determination of erythrocyte PL fatty acids composition and genotyping of single nucleotide polymorphisms were conducted by standard methods. Results: A significant interaction of rs7305618 on HNFIA with C18:2n-6 and C20:4n- 6 was observed: T allele carriers $(\mathrm{TT}+\mathrm{CT}$ ) had a higher risk of T2DM than noncarriers only when they had a higher level of $\mathrm{C} 18: 2 n-6$ or $\mathrm{C} 20: 4 n-6$, and the odds ratios (ORs) were 2.59 (95\% CI 1.58-4.24; $p$ for interaction $=0.005)$ and $2.49(95 \%$ CI 1.47-4.24; $p$ for interaction $=0.021)$, respectively. A significant interaction of rs 8078723 at the intergenic region between PSMD3 and CSF3 with C20: 5n-3 was observed: $C$ allele carriers $(C C+C T$ ) had a lower risk of T2DM than noncarriers only when they had a higher level of C20:5n-3, and the OR was 0.44 ( $95 \%$ CI $0.26-0.73$; p for interaction $=0.014)$. Conclusions: $r s 7305618$ and $r 8078723$ were associated with the risk of T2DM in a Chinese population and were modulated by erythrocyte PL fatty acids composition.
\end{abstract}

(C) 2015 S. Karger AG, Basel

Kelei Li and Tao Huang contributed equally to this study. This paper was presented at the 8th Congress of the International Society of Nutrigenetics/Nutrigenomics (ISNN), Gold Coast, Qld., May 2-3, 2014.

Duo Li

Department of Food Science and Nutrition

Zhejiang University

866 Yu-hang-tang Road, Hangzhou 310058 (China)

E-Mailduoli@zju.edu.cn
Jianqin Sun

Clinical Nutrition Center, Huadong Hospital

Fudan University

Shanghai 200433 (China)

E-Mail jianqins @gmail.com 


\section{Introduction}

Many studies have shown that dietary fat intake was associated with type 2 diabetes mellitus (T2DM) [1]. Our previous case-control study in T2DM subjects and healthy subjects (Chinese) has shown that plasma phospholipid (PL) n-3 polyunsaturated fatty acids (PUFAs) were negatively associated with homeostasis model assessment of insulin resistant (HOMA-IR), blood glucose, and fasting insulin [2]. A meta-analysis of cohort and case-control studies found that marine n-3 PUFA intake was negatively associated with the risk of T2DM in an Asian population [3]. Besides dietary factors, more and more variants in genes have been identified to be associated with T2DM, and some of these variants interact with diet to influence the development of T2DM. Previous studies have shown that variants of FADS, G972R, ADIPOQ, CAV2, TCF7L2, PL1N1, and PPARG had a significant interaction with dietary fat intake on insulin resistance, glucose metabolism or risk of T2DM [4-9]. Our previous study has found that the risk of T2DM was significantly associated with several single nucleotide polymorphisms (SNPs) (rs1801131, rs4646356, and rs2236225) on genes related to homocysteine metabolism in Chinese Hans [10]. In addition, inflammation also plays an important role in the development of T2DM [11,12]. C-reactive protein (CRP) is an acute-phase reactant protein, which is mainly synthesized and secreted by hepatocytes [13]. An elevated CRP level is an independent risk factor for diabetes [14]. Higher white blood cell (WBC) count is associated with a higher risk of T2DM [15]. Previous studies have found many SNPs associated with an elevated inflammation level, such as rs3093059 (CRP), rs2393791 (HNF1A), rs7305618 (HNF1A), rs4537545 (IL6R), rs8078723 (intergenic region between PSMD3 and CSF3), and rs1408282 (gene desert region on chromosome 6q16.1) [16-19]. Our previous meta-analysis of randomized controlled trials showed that marine-derived n-3 PUFA supplementation could significantly reduce blood levels of inflammatory markers, such as CRP, interleukin 6 (IL-6), and tumor necrosis factor $\alpha$ [20]. Therefore, it is reasonable to hypothesize that the protective effect of n-3 PUFAs on T2DM in an Asian population may be attributed to their anti-inflammatory effect. However, no previous study has assessed the interaction between fatty acids and these inflammation-related SNPs.

In the present study, we aimed to assess the interaction of erythrocyte PL fatty acids composition with 6 inflammation-related SNPs (rs3093059, rs2393791, rs7305618, rs4537545, rs8078723, and rs1408282) on the risk of T2DM.

\section{Methods}

Subjects

The subjects in the present study have been described in our previous study [10]. In brief, 622 outpatients with T2DM, who met the WHO criteria for the diagnosis of diabetes mellitus (World Health Organization, 1999), were recruited from 30 hospitals in 20 provinces in China. Subjects were excluded if they received vitamin and mineral supplementation for 3 months, had severe renal, liver, heart, or psychiatric diseases (apart from complications of diabetes), had a history of cancer, thyroid disease, or alcohol abuse, or were pregnant or lactating women. A total of 293 healthy control subjects without hypertension, renal disease, hyperlipidemia, hematological disorders, diabetes, family history of cardiovascular disease and diabetes, or excessive alcohol intake and drug use were recruited through a health check program during the period of March 2011 through October 2011 in Zhejiang Hospital, Hangzhou, China.

The study protocol was approved by the Ethics Committee, College of Biosystem Engineering and Food Science, Zhejiang University, China, and the Institutional Review Board of Huadong Hospital. Written consent has been obtained from all subjects prior to participation in the study.

Determination of Erythrocyte PL Fatty Acids

Venous blood was collected by plain and EDTA vacuum tubes with 21-gauge needles in the morning after an overnight fast. Blood samples were prepared during the $2 \mathrm{~h}$ after the blood had been drawn and were 
Li et al.: Interaction between Erythrocyte Phospholipid Fatty Acids Composition and

Variants of Inflammation-Related Genes on Type 2 Diabetes

aliquoted into separate tubes and stored at $-20^{\circ} \mathrm{C}$ until analysis. The erythrocyte membrane were separated and washed as described in our previous study [21]. The total lipid content of the erythrocyte membrane was extracted by chloroform/methanol (1:1), the PL fraction was separated by thin-layer chromatography, and the fatty acid methyl esters were prepared and separated by gas-liquid chromatography [22]. Data of fatty acid compositions were expressed as weight percentages in total fatty acid methyl esters.

\section{Genetic Analysis}

DNA was isolated from blood samples by QIAamp DNA Blood Mini kits according to the manufacturer's instructions (Qiagen, Valencia, Calif., USA). rs3093059, rs2393791, rs4537545, rs1408282, rs8078723, and rs7305618 have been reported to be associated with inflammation, and they were genotyped by iPlex assay (Sequenom) and MassARRAY Compact System (Sequenom).

\section{Statistical Analysis}

A Hardy-Weinberg equilibrium test for rs3093059, rs2393791, rs4537545, rs1408282, rs8078723, and rs7305618 was conducted by the $\chi^{2}$ test, and an SNP was considered to be consistent with Hardy-Weinberg equilibrium if the $p$ value was $>0.01$ [23]. Data for erythrocyte PL fatty acids composition were expressed as median (interquartile range), because most of them were skewed, and difference was tested by the MannWhitney U test. The association between SNP and T2DM was evaluated by a logistic regression model assuming additive, dominant, and recessive models, adjusted for age and sex. The interactions between erythrocyte PL fatty acids and genotypes on the risk of T2DM were assessed by a logistic regression model, adjusted for age and sex. A p value $<0.05$ was considered to be statistically significant. We also adjusted our primary results, the interaction between 2 SNPs (rs8078723 and rs7305618) and 23 fatty acids on the risk of T2DM, for multiple comparisons (Bonferroni correction). Therefore, a p value $<0.001(0.05 / 2 \times 23)$ was considered significant after multiple comparisons adjustment. All analyses mentioned above were conducted with SPSS 16.0 (Cary, N.C., USA). Data of erythrocyte PL fatty acids content were also imported into SIMCA-P version 13.0 (Umetrics, Umeå, Sweden) for unsupervised principal component analysis (PCA) and the supervised orthogonal partial least squares-discriminant analysis (OPLS-DA). S-plot and variable importance for the projection (VIP) in the OPLS-DA mode were used to find fatty acid markers that contributed most to the discrimination.

\section{Results}

\section{Characteristics of the Subjects and SNPS}

A total of 622 subjects with T2DM (279 males, 342 females) and 293 healthy subjects (149 males, 141 females) were finally included in the present study. The age for the case and control group was $58.97 \pm 11.69$ and $51.09 \pm 13.21$ years, respectively. The minor allele frequency (MAF) was 0.16, 0.39, 0.40, 0.21, 0.46, and 0.47 for rs3093059, rs2393791, rs4537545, rs1408282, rs8078723, and rs7305618, respectively. The MAF of rs3093059, rs2393791, rs4537545, rs1408282, rs8078723, and rs7305618 for Chinese Hans in Beijing in HapMap was $0.17,0.35,0.40,0.18,0.43$, and 0.42 , respectively, and these data were consistent with the results of the present study. All 6 SNPs were consistent with the HardyWeinberg equilibrium test.

\section{Erythrocyte PL Fatty Acids in Healthy Subjects and Subjects with T2DM}

The erythrocyte PL myristoleic acid (C14:1n-5), palmitic acid (C16:0), oleic acid (C18: 1n-7), $\gamma$-linolenic acid (C18:3n-6), arachidic acid (C20:0), and docosapentaenoic acid (C22: $5 n-6)$ were significantly higher in the case group than in the control group. The erythrocyte PL stearic acid (C18:0), linoleic acid (C18:2n-6), eicosatrienoic acid (n-6) (C20:3n-6), erucic acid (C22:1n-9), docosapentaenoic acid (n-3) (C22:5n-3), and docosahexaenoic acid (C22: $6 n-3)$ were significantly lower in the case group than in the control group. No significant difference was observed in other erythrocyte PL fatty acids between the two groups (table 1). 
Li et al.: Interaction between Erythrocyte Phospholipid Fatty Acids Composition and Variants of Inflammation-Related Genes on Type 2 Diabetes

Table 1. Erythrocyte PL fatty acids composition in the case and control groups

\begin{tabular}{lccl}
\hline Fatty acids & Control group & Case group & $\mathrm{p}$ \\
\hline C14:0 & $0.38(0.23,0.57)$ & $0.38(0.21,0.74)$ & $>0.05$ \\
C14:1n-5 & $0.30(0.19,0.58)$ & $0.38(0.22,0.83)$ & $<0.001$ \\
C15:0 & $0.56(0.28,1.03)$ & $0.67(0.36,1.05)$ & $>0.05$ \\
C16:0 & $25.06(22.74,27.39)$ & $26.23(23.40,28.59)$ & $<0.001$ \\
C16:1n-7 & $0.80(0.34,1.21)$ & $0.81(0.56,1.21)$ & $>0.05$ \\
C18:0 & $11.36(10.40,12.36)$ & $9.14(7.82,10.49)$ & $<0.001$ \\
C18:1n-9 & $18.88(9.22,21.86)$ & $18.73(15.35,21.89)$ & $>0.05$ \\
C18:1n-7 & $0.91(0.48,1.19)$ & $1.24(0.90,1.66)$ & $<0.001$ \\
C18:2n-6 & $21.26(18.74,23.94)$ & $19.50(15.11,23.15)$ & $<0.001$ \\
C18:3n-6 & $0.27(0.15,0.51)$ & $0.42(0.20,1.03)$ & $<0.001$ \\
C18:3n-3 & $0.34(0.23,0.60)$ & $0.33(0.19,0.60)$ & $>0.05$ \\
C20:0 & $0.33(0.23,0.57)$ & $0.39(0.23,0.81)$ & $<0.05$ \\
C20:1n-9 & $0.29(0.19,0.48)$ & $0.29(0.19,0.52)$ & $>0.05$ \\
C20:2n-6 & $0.51(0.30,1.05)$ & $0.59(0.27,1.19)$ & $<0.05$ \\
C20:3n-6 & $1.06(0.76,2.20)$ & $0.99(0.64,1.77)$ & $>0.05$ \\
C20:4n-6 & $6.24(4.66,9.71)$ & $6.56(5.08,8.34)$ & $>0.05$ \\
C20:5n-3 & $1.20(0.59,2.27)$ & $1.13(0.71,1.81)$ & $<0.001$ \\
C22:1n-9 & $0.93(0.65,1.33)$ & $0.73(0.39,1.17)$ & $>0.05$ \\
C22:2n-6 & $0.61(0.31,0.92)$ & $0.53(0.29,0.88)$ & $>0.05$ \\
C22:4n-6 & $0.62(0.36,1.13)$ & $0.74(0.44,1.11)$ & $<0.001$ \\
C22:5n-6 & $0.51(0.31,0.79)$ & $0.68(0.40,1.07)$ & $<0.001$ \\
C22:5n-3 & $1.20(0.88,1.52)$ & $0.83(0.52,1.25)$ & $<0.01$ \\
C22:6n-3 & $2.54(1.59,5.07)$ & $2.24(1.54,3.42)$ & \\
\hline
\end{tabular}

Data are medians (interquartile ranges). C14:0 = Myristic acid; C14:1n-5 = myristoleic acid; C15:0 = pentadecanoic acid; C16:0 = palmitic acid; C16:1n-7 palmitoleic acid; C18:0 = stearic acid; C18:1n-9 = oleic acid; C18:1n-7 = vaccenic acid; C18:2n-6 = linoleic acid; C18:3n-6 = $\gamma$-linolenic acid; C18:3n-3 = linolenic acid; C20:0 = arachidic acid; C20:1n-9 = eicosenoic acid; C20:2n-6 = eicosadienoic acid; C20:3n-6 = eicosatrienoic acid (n-6); C20:4n-6 = arachidonic acid; C20:5n-3 = eicosapentaenoic acid; C22:1n-9 = erucic acid; C22: 2n-6 = docosadienoic acid; C22:4n-6 = docosatetraenoic acid; C22:5n-6 = docosapentaenoic acid (n-6); C22: 5n-3 = docosapentaenoic acid (n-3); C22:6n-3 = docosahexaenoic acid.

Healthy subjects and subjects with T2DM could not be clearly discriminated in the 2-dimensional PCA according to the difference in erythrocyte PL fatty acids composition (fig. 1). In order to further characterize their differences, OPLS-DA, a supervised multivariate statistical method to sharpen an already established weak separation between the groups of observations plotted in PCA, was performed after Pareto scaling and mean centering. The 3-dimensional OPLS-DA model showed a clear discrimination between healthy subjects and subjects with T2DM (fig. 2a). In order to reveal potential PL fatty acid markers, S-plot and VIP analysis were constructed following the OPLS-DA. S-plot (fig. 2b) and VIP analysis (data not shown) showed that C18:0, C18:2n-6, and C18:1n-7 made the greatest contribution to the discrimination between healthy subjects and subjects with T2DM by the erythrocyte PL fatty acids profile.

\section{Association between Genotypes and the Risk of T2DM}

rs7305618 showed a significant association with the risk of T2DM under an additive and recessive model: the minor allele $(\mathrm{C})$ homozygotes had a significantly lower risk of T2DM compared with T allele homozygotes (TT) or T allele carriers (TT + CT), and the odds ratios (ORs) were 0.63 (95\% CI 0.41-0.96) and 0.65 (95\% CI 0.46-0.91), respectively. The rs 8078723 
Li et al.: Interaction between Erythrocyte Phospholipid Fatty Acids Composition and Variants of Inflammation-Related Genes on Type 2 Diabetes

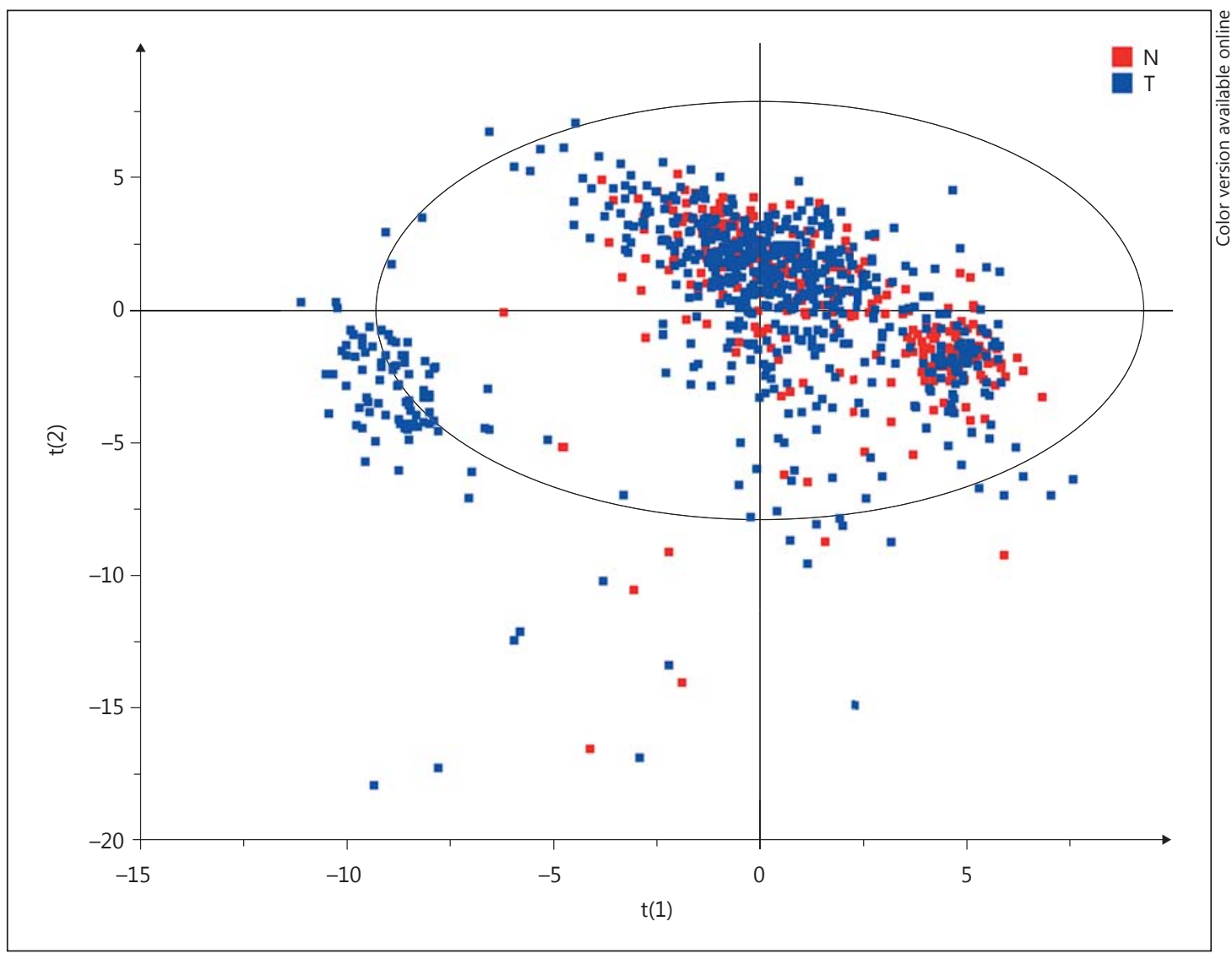

Fig. 1. PCA analysis for erythrocyte PL fatty acids composition in normal subjects and subjects with T2DM. $N=$ Normal subjects $(n=293) ; T=$ subjects with T2DM $(n=622)$.

showed a significant association with the risk of T2DM under the dominant model: the minor allele (C) carriers (CC + CT) had a significantly lower risk of T2DM than noncarriers (TT), and the OR was 0.68 ( $95 \%$ CI 0.48-0.95). No significant result was observed for other SNPs under the three genetic models (table 2).

Interaction between Erythrocyte PL Fatty Acids and Genotypes on the Risk of T2DM

The rs7305618 showed a significant interaction with erythrocyte PL C18:2n-6 and arachidonic acid (C20:4n-6) on the risk of T2DM (p for interaction $=0.005$ and 0.021 , respectively). The major allele (T) carriers (TT $+\mathrm{CT}$ ) had a significantly higher risk of T2DM than noncarriers (CC) only when they had a higher proportion of PL C18:2n-6 or C20:4n-6, and the ORs were 2.59 (95\% CI 1.58-4.24) and 2.49 (95\% CI 1.47-4.24), respectively (table 3).

We also observed a significant interaction between rs8078723 and erythrocyte PL eicosapentaenoic acid $(\mathrm{C} 20: 5 \mathrm{n}-3$; $\mathrm{p}$ for interaction $=0.014)$. The minor allele $(\mathrm{C})$ carriers $(\mathrm{CC}+$ CT) had a significantly lower risk of T2DM than noncarriers (TT) on condition that they had a higher proportion of erythrocyte PL C20:5n-3, and the OR was 0.44 (95\% CI 0.26-0.73; table 3).

After adjusting for multiple comparisons, no significant interaction was observed between genotypes of the two SNPs and fatty acid composition on the risk of T2DM ( $p$ > $0.001)$. 
Li et al.: Interaction between Erythrocyte Phospholipid Fatty Acids Composition and Variants of Inflammation-Related Genes on Type 2 Diabetes
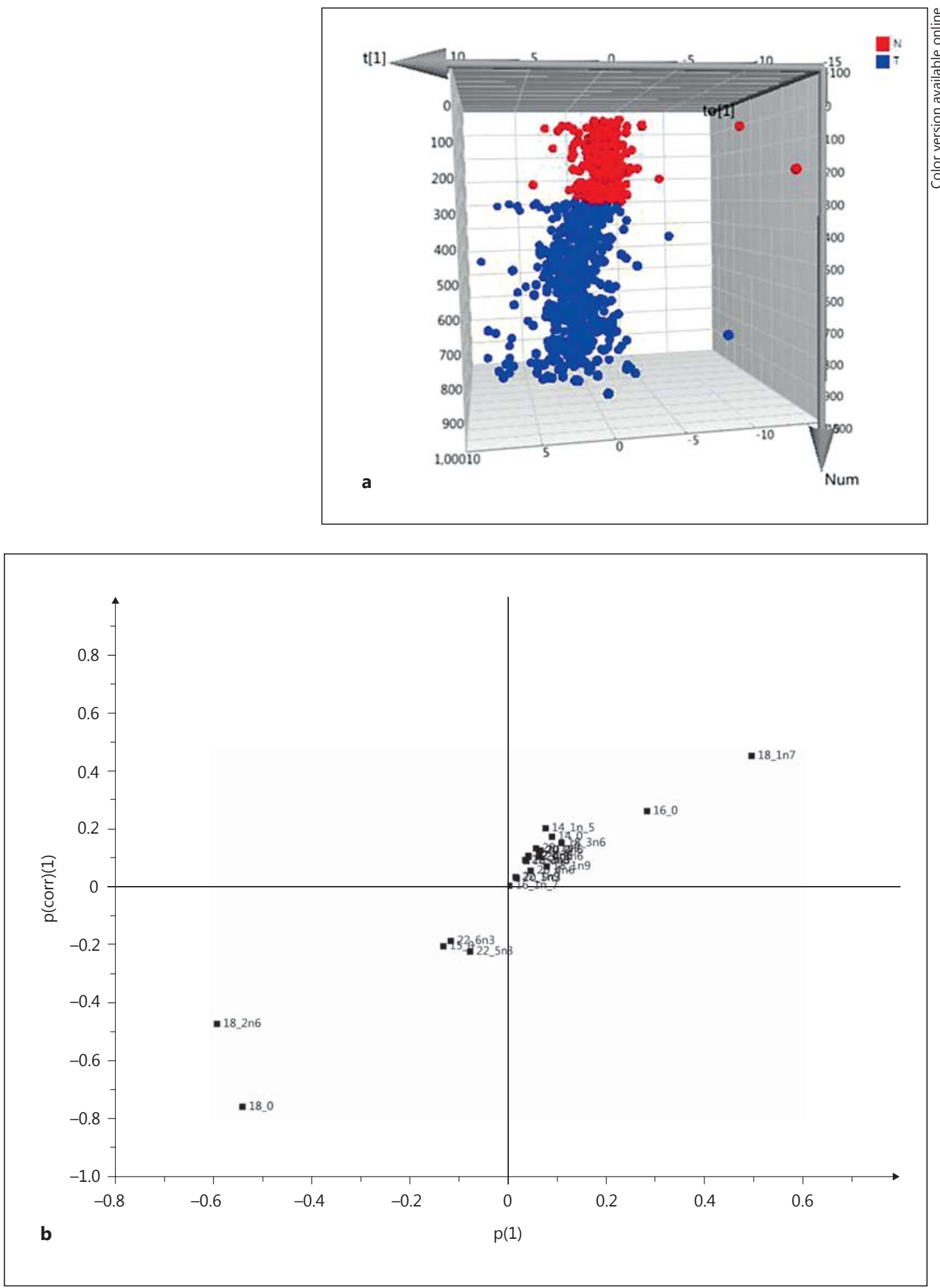

Fig. 2. Three-dimensional OPLS-DA score plot (a) and its corresponding S-plot (b) constructed by erythrocyte PL fatty acids composition in normal subjects and subjects with T2DM. N = Normal subjects $(n=293)$; $\mathrm{T}=$ subjects with T2DM $(\mathrm{n}=622)$. 
Li et al.: Interaction between Erythrocyte Phospholipid Fatty Acids Composition and Variants of Inflammation-Related Genes on Type 2 Diabetes

Table 2. Association between SNPs on inflammation-related genes and the risk of T2DM

\begin{tabular}{|c|c|c|c|c|c|c|}
\hline \multirow[t]{2}{*}{ SNP } & \multicolumn{3}{|l|}{ OR $(95 \% \mathrm{CI})$} & \multirow[t]{2}{*}{$\mathrm{p} 1^{\mathrm{d}}$} & \multirow[t]{2}{*}{$\mathrm{p} 2^{\mathrm{e}}$} & \multirow[t]{2}{*}{$p 3^{f}$} \\
\hline & additive model ${ }^{\mathrm{a}}$ & dominant model ${ }^{b}$ & recessive model $^{\mathrm{c}}$ & & & \\
\hline rs3093059 & $1.01(0.35,2.96)$ & $0.78(0.57,1.06)$ & $1.08(0.37,3.17)$ & 0.984 & 0.116 & 0.883 \\
\hline rs2393791 & $0.72(0.47,1.10)$ & $0.94(0.70,1.27)$ & $0.70(0.48,1.03)$ & 0.129 & 0.693 & 0.068 \\
\hline rs7305618 & $0.63(0.41,0.96)$ & $0.85(0.61,1.18)$ & $0.65(0.46,0.91)$ & 0.032 & 0.330 & 0.013 \\
\hline rs 4537545 & $1.04(0.66,1.63)$ & $1.21(0.90,1.64)$ & $0.92(0.61,1.37)$ & 0.880 & 0.215 & 0.666 \\
\hline rs8078723 & $0.71(0.45,1.13)$ & $0.68(0.48,0.95)$ & $0.95(0.66,1.38)$ & 0.145 & 0.024 & 0.796 \\
\hline rs1408282 & $0.55(0.26,1.16)$ & $0.78(0.58,1.06)$ & $0.60(0.29,1.25)$ & 0.116 & 0.109 & 0.172 \\
\hline
\end{tabular}

Adjusted for age and sex. ${ }^{a}$ Homozygote of minor allele/homozygote of major allele; ${ }^{b}$ minor allele carriers/ noncarriers; ${ }^{\mathrm{c}}$ homozygote of minor allele/major allele carriers; ${ }^{\mathrm{d}}$ for additive model; ${ }^{\mathrm{e}}$ for dominant model; ${ }^{\mathrm{f}}$ for recessive model.

Table 3. Interaction of erythrocyte PL fatty acids composition with rs7305618 and rs8078723 on the risk of T2DM

\begin{tabular}{|c|c|c|c|c|}
\hline \multirow[t]{2}{*}{ Fatty acids } & \multicolumn{2}{|l|}{ rs7305618 } & \multicolumn{2}{|l|}{ rs8078723 } \\
\hline & TT + CT vs. CC & $\mathrm{p}^{\mathrm{a}}$ & $\mathrm{CC}+\mathrm{CT}$ vs. TT & $\mathrm{p}^{\mathrm{a}}$ \\
\hline \multicolumn{5}{|l|}{ C14:0 } \\
\hline Low & $2.06(1.26,3.37)^{* *}$ & 0.149 & $0.54(0.33,0.89)^{*}$ & 0.173 \\
\hline High & $1.23(0.75,2.01)$ & & $0.82(0.51,1.33)$ & \\
\hline \multicolumn{5}{|l|}{ C14:1n-5 } \\
\hline Low & $1.77(1.10,2.84)^{*}$ & 0.446 & $0.57(0.36,0.93)^{*}$ & 0.348 \\
\hline High & $1.34(0.81,2.24)$ & & $0.79(0.49,1.29)$ & \\
\hline \multicolumn{5}{|l|}{ C15:0 } \\
\hline Low & $1.58(0.98,2.54)$ & 0.792 & $0.49(0.30,0.80)^{* *}$ & 0.082 \\
\hline High & $1.43(0.86,2.38)$ & & $0.90(0.56,1.45)$ & \\
\hline \multicolumn{5}{|l|}{ C16:0 } \\
\hline Low & $1.75(1.07,2.86)^{*}$ & 0.462 & $0.54(0.33,0.86)^{* *}$ & 0.221 \\
\hline High & $1.34(0.81,2.20)$ & & $0.84(0.51,1.38)$ & \\
\hline \multicolumn{5}{|l|}{$\mathrm{C} 16: 1 \mathrm{n}-7$} \\
\hline Low & $1.65(0.97,2.79)$ & 0.624 & $0.60(0.36,1.02)$ & 0.67 \\
\hline High & $1.39(0.88,2.21)$ & & $0.72(0.46,1.12)$ & \\
\hline \multicolumn{5}{|l|}{ C18:0 } \\
\hline Low & $1.19(0.59,2.38)$ & 0.348 & $0.80(0.42,1.55)$ & 0.537 \\
\hline High & $1.79(1.11,2.88)^{*}$ & & $0.64(0.41,1.01)$ & \\
\hline \multicolumn{5}{|l|}{ C18:1n-9 } \\
\hline Low & $2.13(1.24,3.66)^{* *}$ & 0.109 & $0.65(0.38,1.10)$ & 0.866 \\
\hline High & $1.19(0.75,1.88)$ & & $0.70(0.45,1.11)$ & \\
\hline \multicolumn{5}{|l|}{ C18:1n-7 } \\
\hline Low & $1.29(0.81,2.04)$ & 0.137 & $0.53(0.34,0.83)^{* *}$ & 0.09 \\
\hline High & $2.34(1.31,4.19)^{* *}$ & & $1.01(0.57,1.81)$ & \\
\hline \multicolumn{5}{|l|}{$C 18: 2 n-6$} \\
\hline Low & $0.92(0.54,1.57)$ & 0.005 & $0.98(0.59,1.61)$ & 0.061 \\
\hline High & $2.59(1.58,4.24)^{* * *}$ & & $0.49(0.31,0.79)^{* *}$ & \\
\hline \multicolumn{5}{|l|}{$C 18: 3 n-6$} \\
\hline Low & $1.34(0.85,2.12)$ & 0.4 & $0.70(0.45,1.11)$ & 0.757 \\
\hline High & $1.81(1.07,3.05)^{*}$ & & $0.63(0.37,1.07)$ & \\
\hline \multicolumn{5}{|l|}{ C18:3n-3 } \\
\hline Low & $1.72(1.07,2.75)^{*}$ & 0.543 & $0.51(0.31,0.85)^{* *}$ & 0.144 \\
\hline High & $1.39(0.83,2.30)$ & & $0.86(0.54,1.38)$ & \\
\hline
\end{tabular}


Li et al.: Interaction between Erythrocyte Phospholipid Fatty Acids Composition and Variants of Inflammation-Related Genes on Type 2 Diabetes

Table 3 (continued)

\begin{tabular}{|c|c|c|c|c|}
\hline \multirow[t]{2}{*}{ Fatty acids } & \multicolumn{2}{|l|}{ rs7305618 } & \multicolumn{2}{|l|}{ rs8078723 } \\
\hline & TT + CT vs. CC & $\mathrm{p}^{\mathrm{a}}$ & $\mathrm{CC}+\mathrm{CT}$ vs. TT & $\mathrm{p}^{\mathrm{a}}$ \\
\hline \multicolumn{5}{|l|}{$\overline{C 20: 0}$} \\
\hline Low & $1.72(1.07,2.77)^{*}$ & 0.537 & $0.69(0.43,1.11)$ & 0.951 \\
\hline High & $1.38(0.83,2.29)$ & & $0.67(0.41,1.10)$ & \\
\hline \multicolumn{5}{|l|}{$\mathrm{C} 20: 1 \mathrm{n}-9$} \\
\hline Low & $1.39(0.85,2.26)$ & 0.47 & $0.65(0.39,1.08)$ & 0.796 \\
\hline High & $1.79(1.09,2.93)$ & & $0.69(0.43,1.10)$ & \\
\hline \multicolumn{5}{|l|}{$C 20: 2 n-6$} \\
\hline Low & $2.00(1.25,3.20)^{* *}$ & 0.124 & $0.68(0.43,1.10)$ & 0.96 \\
\hline High & $1.16(0.69,1.94)$ & & $0.66(0.41,1.08)$ & \\
\hline \multicolumn{5}{|l|}{$C 20: 3 n-6$} \\
\hline Low & $1.27(0.80,2.03)$ & 0.215 & $0.73(0.46,1.16)$ & 0.762 \\
\hline High & $2.01(1.18,3.42)^{* *}$ & & $0.63(0.38,1.05)$ & \\
\hline \multicolumn{5}{|l|}{$C 20: 4 n-6$} \\
\hline Low & $1.07(0.67,1.71)$ & 0.021 & $0.71(0.45,1.12)$ & 0.523 \\
\hline High & $2.49(1.47,4.24)^{* * *}$ & & $0.60(0.35,1.02)$ & \\
\hline \multicolumn{5}{|l|}{$C 20: 5 n-3$} \\
\hline Low & $1.39(0.85,2.28)$ & 0.572 & $1.02(0.64,1.61)$ & 0.014 \\
\hline High & $1.70(1.05,2.77)^{*}$ & & $0.44(0.26,0.73)^{* *}$ & \\
\hline \multicolumn{5}{|l|}{$\mathrm{C} 22: 1 \mathrm{n}-9$} \\
\hline Low & $1.69(1.01,2.82)^{*}$ & 0.672 & $0.74(0.43,1.26)$ & 0.77 \\
\hline High & $1.45(0.90,2.34)$ & & $0.61(0.39,0.97)^{*}$ & \\
\hline \multicolumn{5}{|l|}{$C 22: 2 n-6$} \\
\hline Low & $1.45(0.91,2.32)$ & 0.581 & $0.72(0.45,1.16)$ & 0.824 \\
\hline High & $1,78(1.05,2.99)^{*}$ & & $0.65(0.39,1.08)$ & \\
\hline \multicolumn{5}{|l|}{$C 22: 4 n-6$} \\
\hline Low & $1.49(0.92,2.41)$ & 0.75 & $0.48(0.29,0.78)^{* *}$ & 0.033 \\
\hline High & $1.67(1.01,2.75)^{*}$ & & $0.96(0.60,1.54)$ & \\
\hline \multicolumn{5}{|l|}{$C 22: 5 n-6$} \\
\hline Low & $1.28(0.82,2.01)$ & 0.358 & $0.55(0.35,0.88)^{*}$ & 0.18 \\
\hline High & $1.79(1.04,3.09)^{*}$ & & $0.87(0.52,1.44)$ & \\
\hline \multicolumn{5}{|l|}{$C 22: 5 n-3$} \\
\hline Low & $1.57(0.92,2.69)$ & 0.92 & $0.62(0.35,1.09)$ & 0.777 \\
\hline High & $1.53(0.95,2.48)$ & & $0.67(0.42,1.06)$ & \\
\hline \multicolumn{5}{|l|}{$C 22: 6 n-3$} \\
\hline Low & $1.19(0.73,1.95)$ & 0.093 & $0.71(0.44,1.14)$ & 0.767 \\
\hline High & $2.18(1.32,3.62)^{* *}$ & & $0.63(0.38,1.03)$ & \\
\hline
\end{tabular}

Adjusted for age and sex. C14:0 = Myristic acid; C14:1n-5 = myristoleic acid; C15:0 = pentadecanoic acid; C16:0 = palmitic acid; C16:1n-7 = palmitoleic acid; C18:0 = stearic acid; C18:1n-9 = oleic acid; C18:1n-7 = vaccenic acid; C18:2n-6 = linoleic acid; C18:3n-6 = $\gamma$-linolenic acid; C18:3n-3 = linolenic acid; C20:0 = arachidic acid; C20:1n-9 = eicosenoic acid; C20:2n-6 = eicosadienoic acid; C20:3n-6 = eicosatrienoic acid (n-6); C20:4n-6 = arachidonic acid; C20:5n-3 = eicosapentaenoic acid; C22:1n-9 = erucic acid; C22:2n-6 = docosadienoic acid; C22:4n-6 = docosatetraenoic acid; C22:5n-6 = docosapentaenoic acid (n-6); C22:5n-3 = docosapentaenoic acid (n-3); C22:6n-3 = docosahexaenoic acid.

${ }^{\text {a }} \mathrm{p}$ for interaction. ${ }^{*} \mathrm{p}<0.05$; $^{* *} \mathrm{p}<0.01$; $^{* *} \mathrm{p}<0.001$. 
Li et al.: Interaction between Erythrocyte Phospholipid Fatty Acids Composition and Variants of Inflammation-Related Genes on Type 2 Diabetes

\section{Discussion}

The present study investigated the interaction between erythrocyte PL fatty acids and SNPs of inflammation-related genes on the risk of T2DM (rs3093059, rs2393791, rs7305618, rs4537545, rs8078723, and rs1408282). Several previous studies have assessed the functions of these SNPs in inflammation. One previous study has found that $\mathrm{C}$ allele carriers of rs3093059 had a higher serum level of CRP than noncarriers [24]. Hepatocyte nuclear factor $1 \alpha$ can regulate the expression of CRP by binding to the CRP gene promoter [25]. Two SNPs on HNF1A which encodes hepatocyte nuclear factor $1 \alpha$, rs2393791 and rs7305618, were studied in the present study. Previous studies have shown that the two SNPs were significantly associated with CRP level: C allele carriers of rs2393791 had a lower serum level of CRP than noncarriers [17]; T allele carriers of rs7305618 had a higher plasma level of CRP than noncarriers [19]. The rs4537545, located on IL6R which encodes the IL-6 receptor, had a significant influence on levels of IL- 6 receptor and IL-6: the T allele carriers had a higher level of IL- 6 receptor and IL-6 than noncarriers [16]. A significant association between rs4537545 and CRP level has also been observed: the T allele carriers had a lower plasma level of CRP than noncarriers [16]. The rs8078723 was located at an intergenic region between PSMD3 and CSF3. One previous study found that $\mathrm{C}$ allele carriers of rs8078723 had a higher level of WBCs than noncarriers [17]. As for rs1408282, which is located at a gene desert region on chromosome 6q16.1, a significant association between genotypes and CRP level has been found in one previous study: A allele carriers had a higher plasma level of CRP than noncarriers [19]. No other functions of rs8078723 and rs1408282 have been reported until now.

In the present study, one SNP on HNF1A (rs7305618) and one SNP located at an intergenic region (rs8078723) were identified to have a significant interaction with erythrocyte PL fatty acids on risk of T2DM. HNF1A is a gene encoding hepatocyte nuclear factor 1- $\alpha$, a transcription factor. Previous studies have shown that variants on $H N F 1 A$ were associated with the risk of T2DM $[26,27]$. This association may be attributed to the role which HNF1A plays in inflammation response. Many SNPs on HNF1A have been identified to be associated with the level of CRP, an independent risk factor for T2DM, such as rs7305618, rs2393791, and rs7979473 [28]. In the present study, we found that rs7305618 was associated with the risk of T2DM: the homozygotes of the minor allele (C) had a lower risk of T2DM than T allele carriers (TT + CT). The MAF of rs7305618 in the present study (0.47) was consistent with one previous study (MAF $=0.48$ ), which found a strong association between rs7305618 and plasma CRP levels: the major allele T was positively associated with plasma CRP levels [19].

We observed a significant interaction of rs7305618 with C18:2n-6 and C20:4n-6: the major allele (T) carriers had a significantly higher risk of T2DM than noncarriers only when they had a higher level of erythrocyte PL C18:2n-6 or C20:4n-6. C20:4n-6 is the precursor of eicosanoids, such as prostacyclin $\mathrm{E}_{2}\left(\mathrm{PGE}_{2}\right)$ and leukotriene $\left(\mathrm{LTB}_{4}\right)$ [29]. These eicosanoids have a proinflammatory property, such as modulating the expression of cytokines (e.g. IL-6) [30]. IL-6 plays a key role in the induction of CRP production by hepatocytes. CRP is an independent risk factor for T2DM [14]. C18:2n-6 is an essential fatty acid which can be metabolized into C20:4n-6 in vivo [31]. Therefore, it is possible that $n-6$ PUFAs modulate the expression of CRP and, thus, influence the risk of T2DM. Hepatocyte nuclear factor $1 \alpha$, encoded by the gene $H N F 1 A$, can regulate the expression of CRP by binding to the $C R P$ gene promoter [25]. A mutation of the gene HNF1A may influence the expression of hepatocyte nuclear factor $1 \alpha$ and CRP and, thus, influence the risk of T2DM. Therefore, the modulating effect of $n-6$ PUFAs on the association between rs7305618 and risk of T2DM may be attributed to the influence of n-6 PUFAs on the expression of CRP. Therefore, decreased dietary intake of n-6 PUFAs was recommended. 
Li et al.: Interaction between Erythrocyte Phospholipid Fatty Acids Composition and Variants of Inflammation-Related Genes on Type 2 Diabetes

In the present study, a significant association between rs8078723 and risk of T2DM was observed: the minor allele (C) carriers (CC + CT) had a lower risk of T2DM than noncarriers. The MAF was 0.46 and was consistent with one previous study by Kong and Lee [17] (MAF = $0.47)$. In addition, we also found a significant interaction of rs8078723 with C20:5n-3: the $C$ allele carriers had a lower risk of T2DM only when they had a higher level of C20:5n-3. This result may help explain the mechanism for the effect of n-3 PUFAs on T2DM. Our previous studies have shown that marine-derived n-3 PUFAs had a protective role against T2DM in an Asian population [3]. The anti-inflammatory effect of $n-3$ PUFAs may be one possible mechanism of this protective effect [20]. The study by Kong and Lee [17] found a significant association between genotypes of rs8078723 and WBCs: C allele carriers tended to have a higher WBC count. A previous study has shown that a higher WBC count is associated with a higher risk of T2DM [15]. Therefore, the result of the present study that $C$ carriers had a lower risk of T2DM than noncarriers seems to contradict the study by Kong and Lee [17]. However, the study by Kong and Lee did not assess the association between rs8078723 and the risk of T2DM. One possible reason for this inconsistency is that rs8078723 is located at a region between PSMD3 and CSF3. CSF3 is a gene that encodes colony-stimulating factor 3, a glycoprotein involved in the production, differentiation, and function of granulocytes. Previous studies have shown that colony-stimulating factor 3 can increase WBC count [32]. PSMD3 is a gene that encodes $26 \mathrm{~S}$ proteasome non-ATPase regulatory subunit 3 , which was involved in the ubiquitin-mediated selective protein destruction [33]. Previous studies have shown that the ubiquitin-proteasome pathway plays an important role in insulin signal controlling by means of insulin receptor substrate degradation, insulin receptor ubiquitination, and glucose transporter Glut1 and Glut4 regulation [34]. The rs8078723 had a strong linkage disequilibrium (LD) with rs709592 on PSMD3 $\left(\mathrm{r}^{2}=0.789\right)$, which has been shown to be associated with HOMA-IR and glucose levels in our previous study [23]. rs709592 has been predicted to create a microRNA binding site for hsa-miR-377 [23], which plays an important role in the development of diabetic nephropathy by increasing fibronectin production [35]. Therefore, the association between rs8078723 and risk of T2DM may be attributed to the strong LD between rs8078723 and SNPs on PSMD3, and this may help explain the inconsistency between the present study and the study by Kong and Lee [17]. Previous studies have found that fatty acids can influence the ubiquitin-proteasome pathway and regulate insulin signaling $[36,37]$. Considering the function of PSMD3 in the ubiquitin-proteasome pathway and the strong LD of rs8078723 with the SNP on PSMD3, the modulating effect of fatty acids on the association between rs8078723 and T2DM risk may be attributed to the effect of fatty acids on the ubiquitin-proteasome pathway.

In the present study, no significant association between genotypes and risk of T2DM as well as interaction between genotypes and fatty acid composition on risk of T2DM was observed after multiple-comparisons adjustment (Bonferroni correction). However, considering that a high correlation exists between different fatty acids and between different SNPs, the Bonferroni correction may be too conservative to detect a significant result and may not be suitable in the present study. To increase statistic power, well-designed experiments with a larger sample size are still needed to assess the interaction between fatty acids and the genotypes of these SNPs.

In conclusion, rs7305618 interacts with erythrocyte PL C18:2n-6 and C20:4n-6 to influence the risk of T2DM in a Chinese population: $\mathrm{T}$ allele carriers have a higher risk of T2DM than noncarriers only when they have a higher level of erythrocyte PL C18:2n-6 or C20: $4 n-6$; rs8078723 interacts with erythrocyte PL C20:5n-3 to influence the risk of T2DM in a Chinese population: $\mathrm{C}$ allele carriers have a lower risk of T2DM than noncarriers only when they have a higher level of erythrocyte PL C20:5n-3. In order to reduce T2DM risk, the Chinese population should increase their dietary intake of n-3 PUFA. 
Nournal of
and
Nutrigenetics
Jumics

\section{Acknowledgements}

This work was supported by the National Natural Science Foundation of China (NSFC, No. 81273054), the PhD Programs Foundation of the Ministry of Education of China (20120101110107), and the National Basic Research Program of China (973 Program: 2011CB504002).

\section{Disclosure Statement}

The authors declare that they have no competing interests.

\section{References}

1 Arisawa K, Uemura H, Yamaguchi M, Nakamoto M, Hiyoshi M, Sawachika F, Katsuura-Kamano S: Associations of dietary patterns with metabolic syndrome and insulin resistance: a cross-sectional study in a Japanese population. J Med Invest 2014;61:333-344.

2 Huang T, Wahlqvist ML, Xu TC, Xu A, Zhang AZ, Li D: Increased plasma n-3 polyunsaturated fatty acid is associated with improved insulin sensitivity in type 2 diabetes in China. Mol Nutr Food Res 2010;54:S112-S119.

3 Zheng JS, Huang T, Yang J, Fu YQ, Li D: Marine n-3 polyunsaturated fatty acids are inversely associated with risk of type 2 diabetes in Asians: a systematic review and meta-analysis. PLoS One 2012; 7:e44525.

4 Cormier H, Rudkowska I, Thifault E, Lemieux S, Couture P, Vohl MC: Polymorphisms in fatty acid desaturase (FADS) gene cluster: effects on glycemic controls following an omega-3 polyunsaturated fatty acids (PUFA) supplementation. Genes 2013;4:485-498.

5 Fisher E, Schreiber S, Joost HG, Boeing H, Doring F: A two-step association study identifies CAV2 rs2270188 single nucleotide polymorphism interaction with fat intake in type 2 diabetes risk. J Nutr 2011;141:177-181.

6 Lee YC, Lai CQ, Ordovas JM, Parnell LD: A database of gene-environment interactions pertaining to blood lipid traits, cardiovascular disease and type 2 diabetes. J Data Mining Genomics Proteomics 2011;2:106.

7 Marin C, Perez-Martinez P, Delgado-Lista J, Gomez P, Rodriguez F, Yubero-Serrano EM, Garcia-Rios A, Camargo A, Perez-Jimenez F, Lopez-Miranda J: The insulin sensitivity response is determined by the interaction between the G972R polymorphism of the insulin receptor substrate 1 gene and dietary fat. Mol Nutr Food Res 2011; 55:328-335.

8 Perez-Martinez P, Lopez-Miranda J, Cruz-Teno C, Delgado-Lista J, Jimenez-Gomez Y, Fernandez JM, Gomez MJ, Marin C, Perez-Jimenez F, Ordovas JM: Adiponectin gene variants are associated with insulin sensitivity in response to dietary fat consumption in Caucasian men. J Nutr 2008;138:1609-1614.

9 Ruchat SM, Elks CE, Loos RJF, Vohl MC, Weisnagel SJ, Rankinen T, Bouchard C, Perusse L: Evidence of interaction between type 2 diabetes susceptibility genes and dietary fat intake for adiposity and glucose homeostasis-related phenotypes. J Nutrigenet Nutrigenomics 2009;2:225-234.

10 Huang T, Sun J, Chen Y, Xie H, Xu D, Li D: Associations of common variants in methionine metabolism pathway genes with plasma homocysteine and the risk of type 2 diabetes in Han Chinese. J Nutrigenet Nutrigenomics 2014;7:63-74.

11 Andrews GM, Arredondo OM: Association between ferritin, high sensitivity C-reactive protein (hsCRP) and relative abundance of hepcidin mRNA with the risk of type 2 diabetes in obese subjects. Nutr Hosp 2013;30: 577-584.

12 Brooks-Worrell B, Palmer JP: Immunology in the clinic review series; focus on metabolic diseases: development of islet autoimmune disease in type 2 diabetes patients: potential sequelae of chronic inflammation. Clin Exp Immunol 2012;167:40-46.

13 Yeh ET, Anderson HV, Pasceri V, Willerson JT: C-reactive protein: linking inflammation to cardiovascular complications. Circulation 2001;104:974-975.

14 Freeman DJ, Norrie J, Caslake MJ, Gaw A, Ford I, Lowe GDO, O’Reilly DS, Packard CJ, Sattar N; West of Scotland Coronary Prevention Study: C-reactive protein is an independent predictor of risk for the development of diabetes in the West of Scotland Coronary Prevention Study. Diabetes 2002;51:1596-1600.

15 Gkrania-Klotsas E, Ye Z, Cooper AJ, Sharp SJ, Luben R, Biggs ML, Chen LK, Gokulakrishnan K, Hanefeld M, Ingelsson E, Lai WA, Lin SY, Lind L, Lohsoonthorn V, Mohan V, Muscari A, Nilsson G, Ohrvik J, Chao Qiang J, Jenny NS, Tamakoshi K, Temelkova-Kurktschiev T, Wang YY, Yajnik CS, Zoli M, Khaw KT, Forouhi NG, Wareham NJ, Langenberg C: Differential white blood cell count and type 2 diabetes: systematic review and meta-analysis of cross-sectional and prospective studies. PLoS One 2010;5:e13405.

16 Elliott P, Chambers JC, Zhang W, Clarke R, Hopewell JC, Peden JF, Erdmann J, Braund P, Engert JC, Bennett D, Coin L, Ashby D, Tzoulaki I, Brown IJ, Mt-Isa S, McCarthy MI, Peltonen L, Freimer NB, Farrall M, Ruokonen A, Hamsten A, Lim N, Froguel P, Waterworth DM, Vollenweider P, Waeber G, Jarvelin MR, Mooser V, Scott J, Hall AS, Schunkert H, Anand SS, Collins R, Samani NJ, Watkins H, Kooner JS: Genetic loci associated with C-reactive protein levels and risk of coronary heart disease. JAMA 2009;302:37-48. 


\section{Nutrigenetics Nutrigenomics}

\begin{tabular}{l|l}
\hline J Nutrigenet Nutrigenomics 2014;7:252-263 \\
\hline DOI: 10.1159/000381347 & $\begin{array}{l}\text { @ 2015 S. Karger AG, Basel } \\
\text { www.karger.com/jnn }\end{array}$ \\
\hline
\end{tabular}

Li et al.: Interaction between Erythrocyte Phospholipid Fatty Acids Composition and Variants of Inflammation-Related Genes on Type 2 Diabetes

17 Kong M, Lee C: Genetic associations with C-reactive protein level and white blood cell count in the KARE study. Int J Immunogenet 2013;40:120-125.

18 Mahajan A, Tabassum R, Chavali S, Dwivedi OP, Chauhan G, Ghosh S, Tandon N, Bharadwaj D: Common variants in CRP and LEPR influence high sensitivity C-reactive protein levels in North Indians. PLoS One 2011;6:e24645.

19 Wu Y, McDade TW, Kuzawa CW, Borja J, Li Y, Adair LS, Mohlke KL, Lange LA: Genome-wide association with C-reactive protein levels in CLHNS: evidence for the CRP and HNF1A loci and their interaction with exposure to a pathogenic environment. Inflammation 2012;35:574-583.

20 Li K, Huang T, Zheng J, Wu K, Li D: Effect of marine-derived n-3 polyunsaturated fatty acids on C-reactive protein, interleukin 6 and tumor necrosis factor alpha: a meta-analysis. PLoS One 2014;9:e88103.

21 Huang T, Sun JQ, Chen YQ, Xie H, Xu DF, Huang JY, Li D: Genetic variants in desaturase gene, erythrocyte fatty acids, and risk for type 2 diabetes in Chinese Hans. Nutrition 2014;30:897-902.

22 James AT: Qualitative and quantitative determination of the fatty acids by gas-liquid chromatography. Methods Biochem Anal 1960;8:1-59.

23 Zheng JS, Arnett DK, Parnell LD, Lee YC, Ma YY, Smith CE, Richardson K, Li D, Borecki IB, Ordovas JM, Tucker KL, Lai CQ: Genetic variants at PSMD3 interact with dietary fat and carbohydrate to modulate insulin resistance. J Nutr 2013;143:354-361.

24 Wang Q, Hunt SC, Xu Q, Chen YE, Province MA, Eckfeldt JH, Pankow JS, Song Q: Association study of CRP gene polymorphisms with serum CRP level and cardiovascular risk in the NHLBI family heart study. Am J Physiol Heart Circ Physiol 2006;291:H2752-H2757.

25 Li SP, Goldman ND: Regulation of human C-reactive protein gene expression by two synergistic IL-6 responsive elements. Biochemistry 1996;35:9060-9068.

26 Morita K, Saruwatari J, Tanaka T, Oniki K, Kajiwara A, Otake K, Ogata Y, Nakagawa K: Associations between the common HNF1A gene variant p.I27l (rs1169288) and risk of type 2 diabetes mellitus are influenced by weight. Diabetes Metab 2015;41:91-94.

27 Wakil SM, Muiya NP, Tahir AI, Al-Najai M, Baz B, Andres E, Mazhar N, Al Tassan N, Alshahid M, Meyer BF, Dzimiri N: A new susceptibility locus for myocardial infarction, hypertension, type 2 diabetes mellitus, and dyslipidemia on chromosome 12q24. Dis Markers 2014;2014:291419.

28 Reiner AP, Barber MJ, Guan Y, Ridker PM, Lange LA, Chasman DI, Walston JD, Cooper GM, Jenny NS, Rieder MJ, Durda JP, Smith JD, Novembre J, Tracy RP, Rotter JI, Stephens M, Nickerson DA, Krauss RM: Polymorphisms of the HNF1A gene encoding hepatocyte nuclear factor-1 alpha are associated with C-reactive protein. Am J Hum Genet 2008;82:1193-1201.

29 Williams K, Higgs G: Eicosanoids and inflammation. J Pathol 1988;156:101-110.

30 Calder PC: n-3 polyunsaturated fatty acids, inflammation, and inflammatory diseases. Am J Clin Nutr 2006; 83:S1505-S1519.

31 Wallis JG, Watts JL: Polyunsaturated fatty acid synthesis: what will they think of next? Trends Biochem Sci 2002;27:467-473.

32 Brender E, Lynm C, Glass RM: Granulocyte-colony stimulating factor. JAMA 2006;295:1088.

33 Glickman $\mathrm{MH}$, Ciechanover A: The ubiquitin-proteasome proteolytic pathway: destruction for the sake of construction. Physiol Rev 2002;82:373-428.

34 Rome S, Meugnier E, Vidal H: The ubiquitin-proteasome pathway is a new partner for the control of insulin signaling. Curr Opin Clin Nutr Metab Care 2004;7:249-254.

35 Wang Q, Wang Y, Minto AW, Wang J, Shi Q, Li X, Quigg RJ: Microrna-377 is up-regulated and can lead to increased fibronectin production in diabetic nephropathy. FASEB J 2008;22:4126-4135.

36 Hamel FG, Fawcett J, Bennett RG, Duckworth WC: Control of proteolysis: hormones, nutrients, and the changing role of the proteasome. Curr Opin Clin Nutr Metab Care 2004;7:255-258.

37 Yu C, Chen Y, Cline GW, Zhang D, Zong H, Wang Y, Bergeron R, Kim JK, Cushman SW, Cooney GJ: Mechanism by which fatty acids inhibit insulin activation of insulin receptor substrate-1 (irs-1)-associated phosphatidylinositol 3-kinase activity in muscle. J Biol Chem 2002;277:50230-50236. 